\title{
Factores que influyen en el uso de métodos anticonceptivos modernos en la población panameña sexualmente activa entre los 15-59 años de edad en el año 2009
}

\author{
Lorna Jenkins ${ }^{1, *}$, Ilenia Forero ${ }^{2}$ y John Hembling \\ ${ }^{1}$ Oficina Regional de Latinoamérica y el Caribe, Fondo de Población de las Naciones Unidas. \\ ²Oficina de Planificación, Hospital del Niño, Panamá, República de Panamá. \\ *Autor para correspondencia. Email: lienkins@unfpa.org
}

Recibido: 17 de noviembre de 2014

Aceptado: 5 de diciembre de 2014

\begin{abstract}
Introduction. The present study aims to explore factors that might be associated with a SRH indicator such as the use of modern contraceptives in sexually active men and women.

Materials and Methods. This is a secondary data analysis from the national sexual and reproductive health survey, conducted in 2009. The survey used the Census of Population and Housing conducted in 2000 as the sample frame. Analyses were performed using the statistical analysis program Stata SE 13.0.

Results. The use of modern contraceptives is statistically significant in non-indigenous men and women and those who live in urban areas. Educational attainment, being sexually active before age 18 and having more than two sexual partners in a year were statistically significant with the condom use in men and were not associated with the use of modern contraceptives in women.

Conclusions. Efforts are needed to ensure access to sexual and reproductive health services for women, those who are living in indigenous areas, and persons self-identified as indigenous.
\end{abstract}

Keywords: Modern contraceptives; Contraception; Sexual and Reproductive Health; Indigenous; Access to health services.

\section{Resumen}

Introducción. El presente estudio tiene como propósito estudiar los factores que potencialmente se pueden asociar a un indicador de SSR como es el uso de métodos anticonceptivos modernos en hombres y mujeres con vida sexual activa. 
Invest. pens. crit.

Vol. 2, No. 5, septiembre-diciembre 2014.

pp. $51-62$

Materiales y métodos. Es un análisis secundario a partir de la Encuesta Nacional de Salud Sexual y Reproductiva 2009 que utilizó como marco de muestreo el Censo de Población y Vivienda realizado en el año 2000. Los análisis se realizaron con el programa de análisis estadístico Stata SE 13.0.

Resultados. El uso de anticonceptivos modernos es estadísticamente significativo tanto en hombres como en mujeres no indígenas y que viven en áreas urbanas. La escolaridad, haber iniciado relaciones sexuales antes de los 18 años y tener más de dos parejas sexuales en el año se asoció de manera estadísticamente significativa al uso del condón en hombres y no se asoció al uso de anticonceptivos modernos en mujeres.

Conclusiones. Profundizar los esfuerzos para garantizar el acceso a servicios de salud sexual y reproductiva para las mujeres, en las áreas indígenas, y a la población auto-identificada como indígena.

Palabras clave: Anticonceptivos modernos; Anticonceptivos; Salud sexual y reproductiva; Indígenas; Acceso a servicios de salud

\section{Introducción}

Panamá es el país de América Latina con el crecimiento económico sostenido más alto en el periodo 2008-2012 (World Economic Forum [WEF], 2013), está situado en la posición número 40 de 144 países en cuanto a competitividad global se refiere. A pesar de ser un país con indicadores económicos favorables, los indicadores de salud no reflejan este auge económico.

La población de Panamá es de 3,405,813 habitantes (Contraloría General de la República de Panamá [CG], 2011), de los cuales el 17\% se encuentran entre 15-24 años y el 52\% son mujeres en edad reproductiva, es decir entre 10-49 años. Los indicadores de salud sexual y reproductiva han mejorado discretamente, tal es el caso de la mortalidad materna que ha variado de 61.7 a 60.3 por 100,000 nacidos vivos en los últimos diez años (Organización Panamericana de la Salud [OPS], 2013); la tasa global de fecundidad se ha modificado de 2.7 a 2.4 por cada 1000 mujeres en edad reproductiva (Banco Mundial, 2013); y la tasa específica de fecundidad en adolescentes se encuentra en 88/1000 mujeres con edades entre 15-19 años (Organización Mundial de la Salud [OMS], 2014).

En Panamá mediante la Encuesta Nacional de Salud Sexual y Reproductiva (ENASSER) en 2009 se recolectaron variables relevantes para la salud sexual y reproductiva, como es el caso del uso de métodos anticonceptivos modernos, entre otros.

En la literatura, los factores facilitadores descritos para el uso de métodos anticonceptivos modernos son:

- Área de residencia. Se considera que el vivir en área rural se asocia con una actitud menos favorable para el uso de anticonceptivos modernos (De La Cruz-Peñarán, et al., 2003), siendo una de sus características el tener difícil acceso (González, 2008). 
- Nivel de escolaridad. Hombres y mujeres con nivel secundario presentan un uso más bajo de métodos anticonceptivos (Vásquez \& Suárez, 2005; Fétis, et al., 2010).

- Inicio de vida sexual. A pesar de que pueda existir un inicio temprano de vida sexual, estos sujetos tienen conocimientos sobre diversos métodos anticonceptivos y refieren su uso, más del $80 \%$, en la primera relación sexual (Reis, Ramiro, Gaspar \& Alves, 2012; Trejo-Ortiz, et al., 2011; Fétis, et al., 2010).

- Religión. Indistintamente de la religión que predomine en un grupo o población estudiado, ésta no parece afectar al momento de la toma de decisiones en cuanto al uso de anticonceptivos modernos (Vásquez \& Suárez, 2005; Fétis, et al., 2010).

- Número de parejas sexuales. El hecho de tener dos o más parejas sexuales conlleva al uso de dos o más métodos anticonceptivos de manera simultánea (Carrasco-Garrido, et al., 2011).

El objetivo principal de este análisis secundario es conocer los factores que influyen en el uso de métodos anticonceptivos modernos entre los hombres y mujeres sexualmente activos que participaron en ENASSER 2009. Este conocimiento es importante para poder adaptar y diseñar estrategias basadas en evidencia con el propósito de mejorar los indicadores de salud relacionados con la salud sexual y reproductiva como lo son la fecundidad, embarazos en adolescentes y la mortalidad materna. Estos temas se consideran en deuda con los objetivos del milenio y cobran más protagonismo de cara a la construcción de la agenda post 2015, ahora en un contexto más amplio, con el enfoque de derechos humanos, equidad en el acceso, con la perspectiva del cuidado continuo de la vida, y libertad de tomar decisiones informadas por una persona, una pareja o una familia.

\section{Materiales y métodos}

\section{Muestra}

La ENASSER de 2009 es la primera encuesta nacional dedicada al tema de salud sexual y reproductiva. El marco de muestreo para esta encuesta estuvo basado en el X Censo de Población y VI de Vivienda de la República de Panamá del año 2000.

Para esta encuesta la unidad primaria de muestreo es el segmento censal; la unidad secundaria de muestreo es la vivienda y la unidad de observación corresponde a las mujeres en el rango de 15 a 49 años de edad, que se encuentren en los dos primeros segmentos seleccionados y los hombres de 15 a 59 años de edad que se encuentren en cada tercer segmento seleccionado.

\section{Definición de variables}

Diversos factores han mostrado algún grado de influencia en el uso de anticonceptivos modernos y en hombres y mujeres. Para entender mejor estas variables (dependientes) las definiremos como: 
Invest. pens. crit.

Vol. 2, No. 5, septiembre-diciembre 2014.

pp. $51-62$

- Utilización de métodos anticonceptivos modernos: uso de productos o intervenciones quirúrgicas que afectan la capacidad reproductiva de un individuo, o una pareja en forma temporal o permanente cuando tienen relaciones sexuales. Estos métodos pueden ser hormonales (inyectables e implantes), de barrera (dispositivo intrauterino y condón) y cirugías tanto masculina como femenina (Vásquez \& Suárez, 2006). En este caso, la utilización de métodos anticonceptivos modernos en hombres se refiere a la utilización de condón y en mujeres, se define como el uso de métodos hormonales o de barrera alguna vez previo a la aplicación de la encuesta.

Los factores que consideramos importante mencionar para determinar si presentan alguna asociación el uso de anticonceptivos modernos son los siguientes:

- Etnia. Es una comunidad humana definida por afinidades raciales, lingüísticas, culturales (Real Academia de la Lengua Española [RAE]). Para efectos de este análisis los individuos serán clasificados como no indígenas, que incluye a todos aquellos que no se consideren indígenas o de algún otro grupo étnico, o indígenas, que incluye los 9 grupos existentes en $\mathrm{Pa}$ namá (Instituto Nacional de Estadísticas y Censo [INEC], 2010).

- Área de residencia. Se refiere a una localidad separada físicamente de otra (INEC, 2010) y que según las características de las viviendas y el acceso a bienes. Se clasificó en urbano, rural e indígena.

- Nivel de escolaridad. Se refiere al grado o año escolar más alto aprobado dentro de los niveles que se imparten en escuelas y universidades públicas y privadas del país (INEC, 2010). Para efectos del análisis se agruparon en: sin escolaridad o primaria, hasta secundaria y con educación superior.

- Religión. Es el conjunto de creencias o dogmas acerca de la divinidad, de normas morales para la conducta individual y social y de prácticas rituales (RAE). Según las religiones establecidas en el país tomadas en cuenta para esta encuesta se agruparon en: católica, cristiana, otra o ninguna.

- Inicio de vida sexual. Para fines de este análisis se definió como antes o a los 15 años de edad, entre los 16 y 17 años de edad, o a los 18 o más años de edad.

- Número de parejas sexuales en los últimos 12 meses. Para fines de este análisis se definió agrupando las respuestas según 1 o menos parejas, o respondiendo 2 o más parejas. Esta agrupación se definió tomando en consideración todas las respuestas posibles dadas por los encuestados y buscando la media de la misma.

\section{$\underline{\text { Análisis estadístico }}$}

Los datos para el análisis secundario presentado a continuación provienen de los resultados de la encuesta ENASSER 2009. A través de la revisión de los resultados de la encuesta y la base de datos se identificaron las variables dependientes e independientes que deseamos estudiar. Dicho análisis se realizó con el programa informático de análisis estadístico Stata SE 13.0. 
Una vez logramos ubicar las variables y agruparlas según la necesidad, realizamos las asociaciones correspondientes para determinar si existían diferencias. Estas diferencias fueron analizadas con Chi cuadrado de Pearson para comprobar si dichas diferencias eran estadísticamente significativas. En los casos en que la variable independiente tenía más de 2 categorías, se utilizó el Test de Wald para encontrar en cuales categorías existía la diferencia estadística.

\section{$\underline{\text { Limitaciones }}$}

Por ser este un análisis secundario presentamos las siguientes limitaciones:

- Análisis de una encuesta transversal. Sólo se pudieron examinar asociaciones entre las variables previamente definidas seleccionadas con base en nuestro interés, es decir, no permitió realizar un análisis de causalidad.

- La metodología de recolección de los datos. La encuesta se realizó cara a cara y los sujetos debían auto-reportar conductas sexuales, por lo que puede existir sesgo sistemático en las respuestas.

- $\quad$ El análisis bivariado también constituye una limitante en el presente análisis. Este tipo de análisis permite realizar un tratamiento estadístico a dos variables, pero no podemos estimar si otra variable además de estas primeras dos, influye en tal asociación.

\section{Resultados}

Características de la población estudiada.

Para este análisis se seleccionó del universo participante en la encuesta ENASSER 2009 aquella población conformada por los hombres entre 15 y 59 años de edad y las mujeres entre 15 y 49 años de edad que reportaran haber iniciado vida sexual.

De los 2831 hombres entre 15 y 59 años de edad encuestados $2456(86.8 \%)$ reportaron haber iniciado vida sexual, y de las 5831 mujeres entre 15 y 49 años de edad encuestados 4903 (84.1\%) reportaron igual condición, para una población total de 7359 personas con vida sexual. Un $68 \%$ de estos hombres y un $69.4 \%$ de estas mujeres vivían en áreas urbanas, mientras que el 32\% de estos hombres y 30.6\% de estas mujeres se concentraban en las áreas rural y comarcas. El 89.5\% de los hombres y el 90.1\% de las mujeres no pertenecían a ningún grupo indígena.

Es notable que el $52.1 \%$ y $48.9 \%$ de hombres y mujeres completaron hasta secundaria, así como un $19.8 \%$ y $24.8 \%$ de hombres y mujeres llegaron a una educación superior. El mayor grupo religioso reportado fue el católico con un $74.5 \%$ en los hombre y $70.4 \%$ en las mujeres. 
Invest. pens. crit.

Vol. 2, No. 5, septiembre-diciembre 2014.

pp. $51-62$

En cuanto a la sexualidad, un $75.6 \%$ de los hombres y $51.4 \%$ de las mujeres reportaron haber iniciado vida sexual antes de los 18 años de edad. Tanto en el grupo de hombres como en el de mujeres predominó el reporte de 1 o menos parejas sexuales en los últimos 12 meses con $75.5 \%$ y $92.2 \%$ respectivamente.

En relación al uso de anticonceptivos modernos el 71.1\% de los hombres reportaron haber usado alguna vez el condón, y de estos solo 15.2\% lo utilizan con frecuencia. Mientras que el 53.8\% de las mujeres refiere estar utilizando algún método anticonceptivo en la actualidad.

Figura 1.Utilización del condón o métodos anticonceptivos modernos alguna vez en según área de residencia $(p<0.05)$

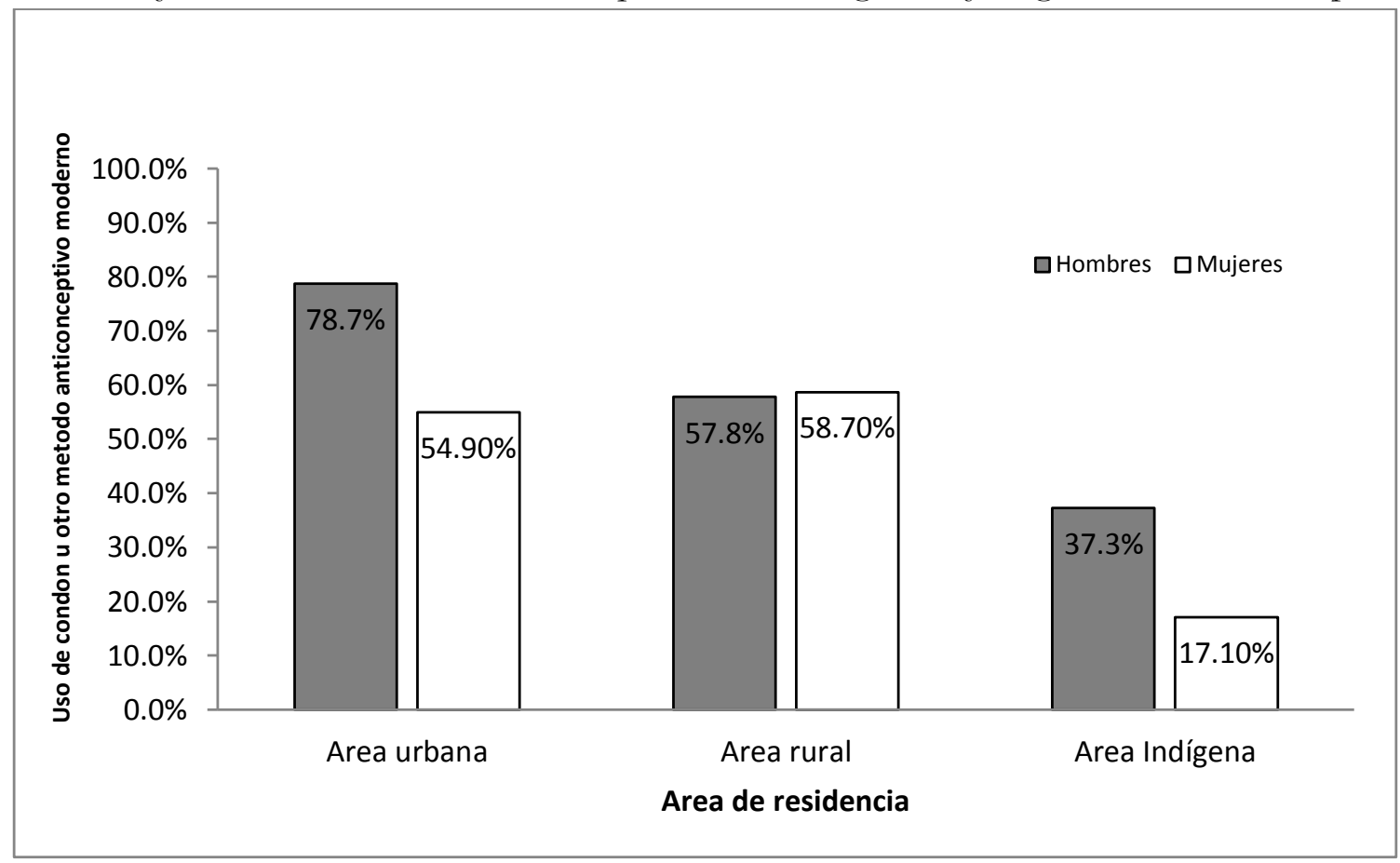

$\underline{\text { Factores asociados al uso de anticonceptivos modernos }}$

En los hombres sexualmente activos encontramos que entre los no indígenas el 75.1\% y solo el 36.8\% del grupo de hombres indígenas refieren uso de condón $(\mathrm{p}<0.05)$. Al analizar la utilización de métodos anticonceptivos modernos en las mujeres sexualmente activas encontramos que el $57.1 \%$ de las mujeres no indígenas y el 23.3\% de las mujeres indígenas los usaban $(\mathrm{p}<0.05)$.

Según la distribución por área de residencia encontramos que un 78.7\% de los hombres residentes en áreas urbanas utilizan el condón, mientras quienes residen en área rural los utiliza en el 57.8\% y 37.3\% de los que habitaban en área indígena $(\mathrm{p}<0.05)$. Mientras que las mujeres que residen en áreas rurales reportaron el uso de anticonceptivos modernos en un 58.7\%, comparado con el 54.9\% de las que residen en área urbana y un 17.1\% de las que residen en el área indígena ( $\mathrm{p}<0.05)$. (Figura 1). 
En relación al uso de condón según nivel de escolaridad, se encontró que el 78.1\% de los hombres con estudios de secundaria y el $88.6 \%$ con una educación superior lo utilizaban algunas veces. Mientras que entre el grupo de hombres sin escolaridad o que sólo acudieron hasta la primaria la utilización de condón fue de $45.8 \%$. Todas estas asociaciones resultaron estadísticamente significativas con $\mathrm{p}<0.001$. (Figura 2). Mientras que en las mujeres, indistintamente del nivel de escolaridad, más del $50 \%$ de las mujeres en cada grupo reportaron la utilización de algún método anticonceptivo moderno, siendo esta asociación no estadísticamente significativa.

Figura 2. Utilización del condón en hombres de 15 a 59 años de edad según nivel de escolaridad $(p<0.05)$

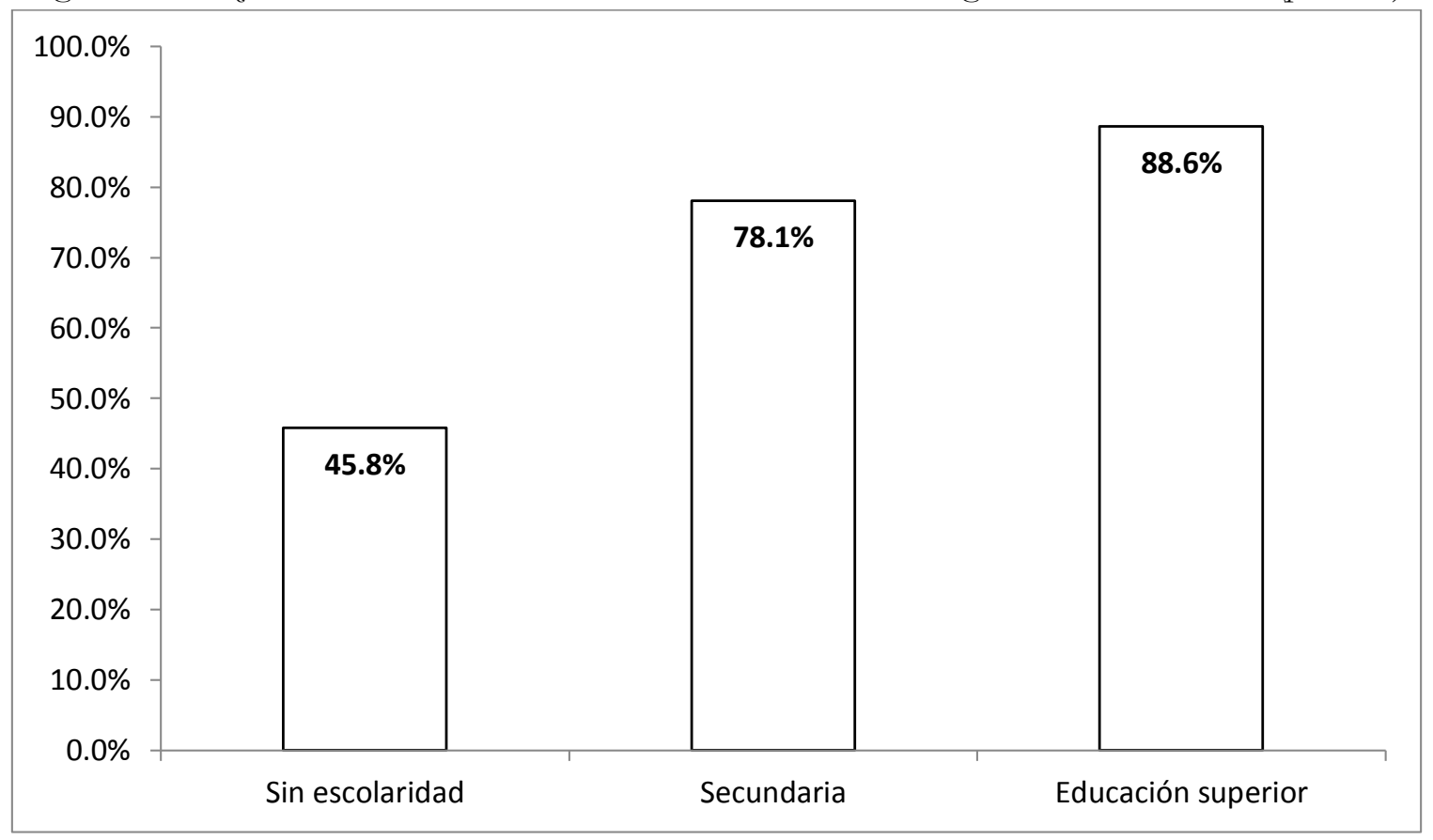

Cabe mencionar que un grupo importante de los que iniciaron relaciones sexuales antes de los 18 años, $78.6 \%$ de los $\leq 15$ años y 72.4\% de los que estaban entre 16 y 17 años, afirmaron usar condón, contrario a lo observado en los que iniciaron actividad después de los 18 años donde se hacía con menor frecuencia con un 59.7\%. Asociación que es estadísticamente significativa con una p de 0.0006. (Figura 3). Al comparar la edad de inicio de vida sexual de las mujeres con el uso de métodos anticonceptivos modernos no se encontró asociación estadísticamente significativa. 
Invest. pens. crit.

Vol. 2, No. 5, septiembre-diciembre 2014.

pp. $51-62$

Figura 3. Utilización del condón en hombres de 15 a 59 años de edad según edad de inicio de vida sexual ( $p<0.05)$

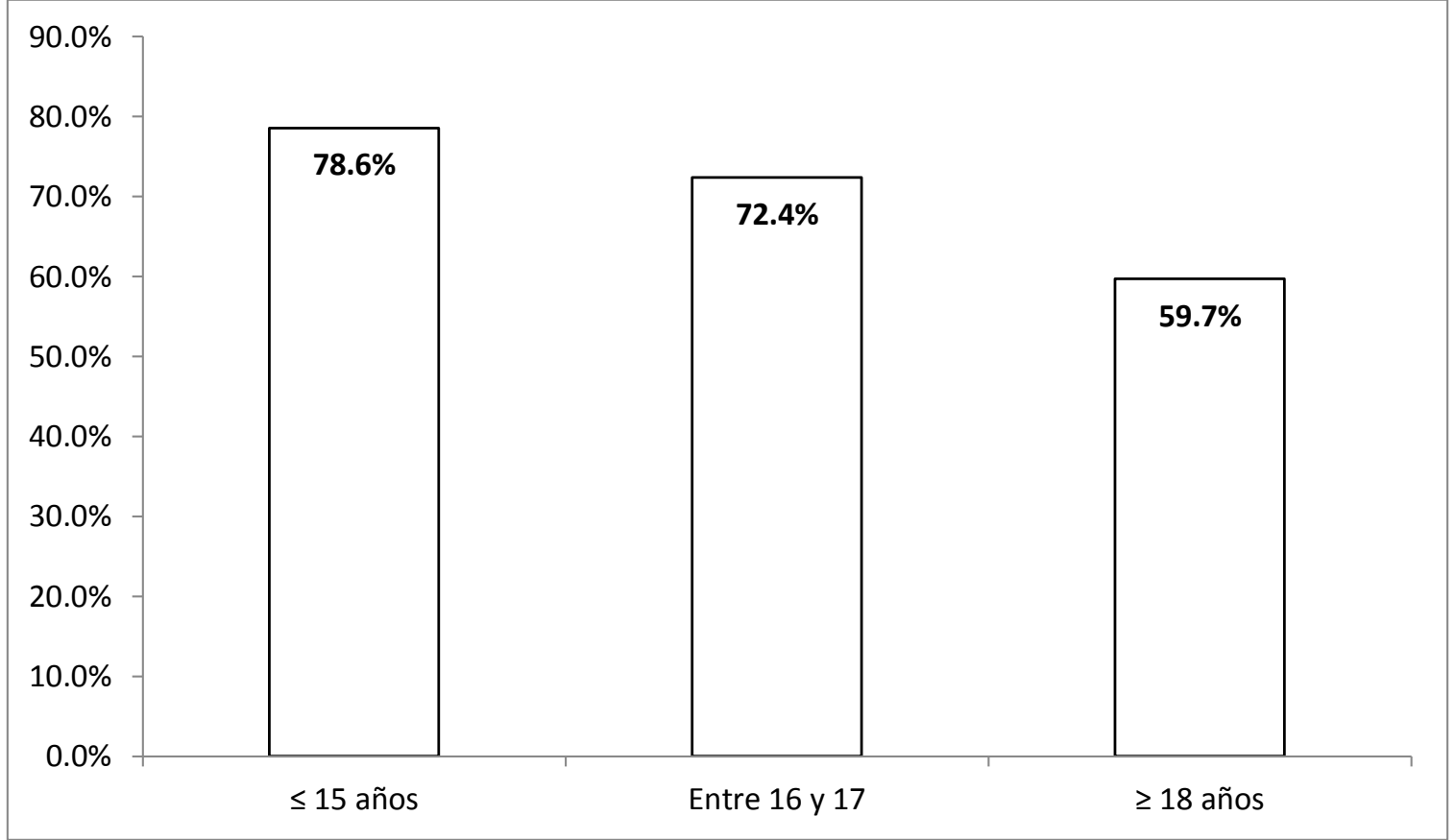

En relación al número de parejas sexuales en los doce meses previo a la encuesta, el 87.8\% de los hombres con 2 o más parejas sexuales afirmaron usar el condón. Igualmente el 65.6\% de los hombres con 1 pareja sexual o menos. Esta diferencia resultó estadísticamente significativo con una p $<0.001$. (Figura 4). Entre las mujeres el número de parejas sexuales en los últimos 12 meses no mostro asociación estadísticamente significativa. Es decir, el número de parejas no influye en el uso de métodos anticonceptivos modernos.

\section{Discusión}

Para una mejor visualización de los resultados según el análisis estadístico de las variables, hemos agrupado los mismos en tres ideas principales que reflejan los factores que influyeron en la decisión de la utilización de dichos métodos anticonceptivos modernos.

Garantizar el acceso a servicios de salud sexual y reproductiva así como el uso de anticonceptivos modernos en áreas indígenas y en población auto-identificada como indígena

El análisis reveló brechas en la utilización de métodos anticonceptivos tanto en mujeres como en hombres identificados como indígenas, lo que fue estadísticamente significativo; al igual que entre las áreas de residencia indígena, al compararlas con urbana y rural. El uso de condón entre hombres indígenas es de tan solo 36.8\%, mientras que en el no indígena es de 75.1\%; entre los hombres que viven en áreas indígenas el uso de condón es de tan solo $37.3 \%$, en las áreas urbanas y rural su uso está arriba del 50.0\%. Entre las mujeres indígenas la utilización de métodos anticonceptivos modernos es de $23.3 \%$ y en las no indígenas es de $57.1 \%$. El 17.0\% de las mujeres que viven en áreas indígenas 
reportaron su uso, mientras que en las áreas urbana y rural el uso supera el 50\%. Estos hallazgos son similares a los de Taylor, Hembling y Bertrand (2014) donde la etnia indígena con un bajo porcentaje de representación en el área urbana reportó el menor conocimiento y uso de servicios de salud sexual y reproductiva, debido en parte al limitado acceso a los servicios de salud, consejería y oportunidad de realización de la prueba.

Figura 4. Utilización del condón en hombres de 15 a 59 años de edad según número de parejas sexuales $(p<0.05)$

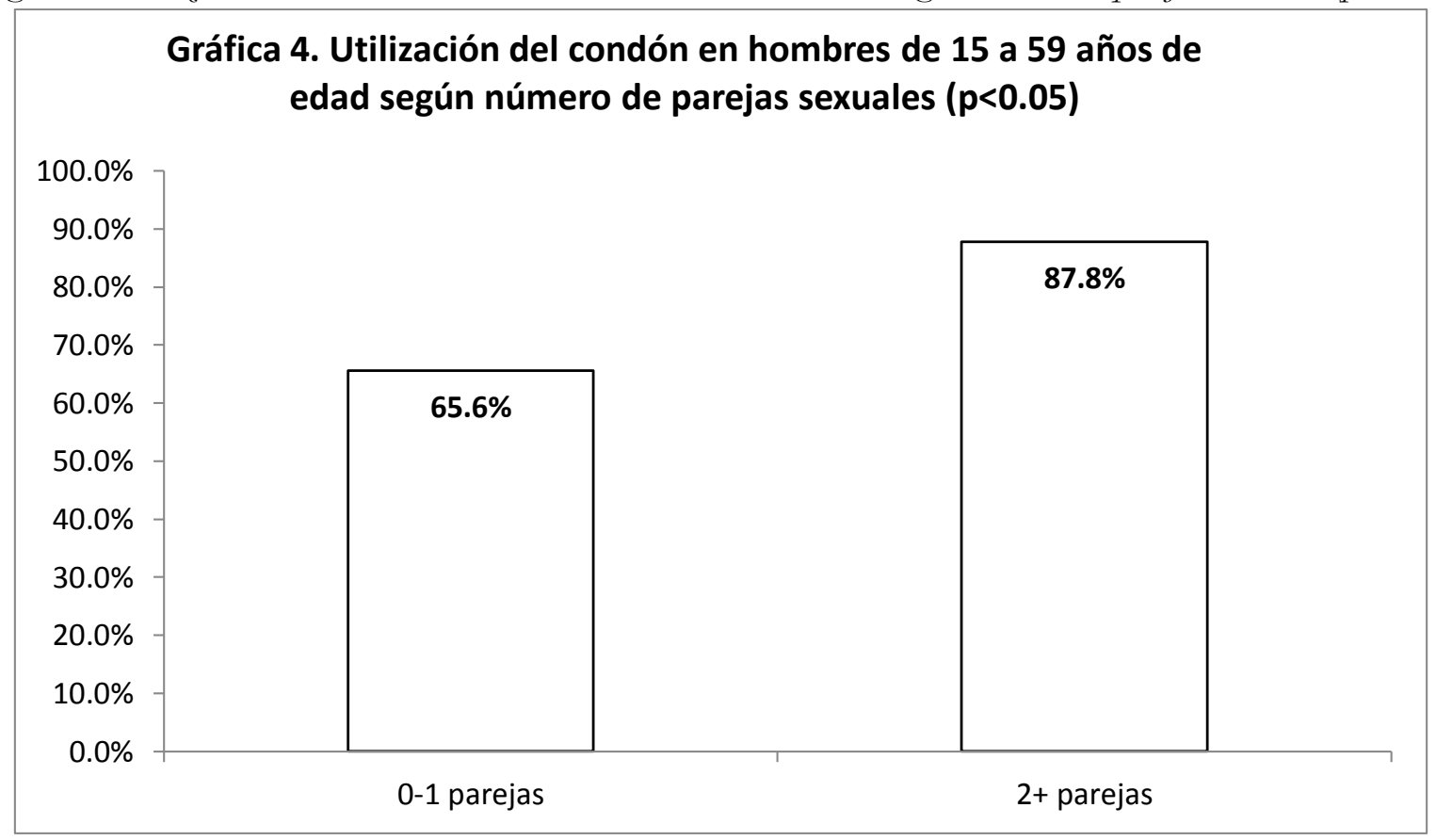

Evitar la deserción escolar e incluir a los hombres en las estrategias

Alcanzar la educación primaria universal, es pilar para el desarrollo integral y sostenible de un país una vez que se inculca la necesidad e importancia de la educación. Varios estudios mencionan la educación como un factor facilitador para el uso de anticonceptivos. El estudio de Vásquez y Suárez (2005) reporta que entre 61 a 77\% de los que cuentan con un título de técnico o bachillerato refieren uso de métodos anticonceptivos modernos sin hacer diferencia en cuanto al sexo. De igual forma en el informe chileno sobre la situación de salud y derechos en salud sexual y reproductiva (2003) se indica que a mayor escolaridad en ambos sexos, desde secundaria a profesionistas, mayor uso de anticonceptivos lo que va desde 50 a 62\%. En Panamá el nivel de escolaridad no se asoció con el uso de anticonceptivos modernos en las mujeres, pero si está asociado con significancia estadística el uso del condón en varones con educación superior. Recomendamos continuar los esfuerzos para evitar la deserción escolar, en especial de los hombres, y su inclusión en los temas de salud sexual y reproductiva.

\section{Educación sexual desde temprana edad para ambos sexos.}

El análisis encontró que el inicio de la actividad sexual en Panamá es antes de los 18 años (75.6\% en los hombres y $51.4 \%$ en las mujeres). Esto refuerza la urgencia de establecer como una política de 
Invest. pens. crit.

Vol. 2, No. 5, septiembre-diciembre 2014.

pp. $51-62$

estado la educación integral de la sexualidad tanto a nivel escolar como comunitario. Estudios muestran que la fuente de la información es importante al momento de medir la efectividad en el uso del método escogido (Clifton, Brice, Wiggins-Smith, Penrose \& Farooqi, 2013). Al realizar el análisis con respecto a grupos de inicio de relaciones sexuales por edad, en el grupo de menos de 15 años fue más frecuente el uso de condón en los hombres, mientras que en las mujeres la edad no influye en el uso de anticonceptivos modernos; contrario a lo encontrado en el estudio realizado por Vásquez y Suárez (2005) en el que el grupo de 15-19 años son los que reportaron el menor uso de anticonceptivos con un $57.1 \%$, mientras que los de 20 a 44 años reportaron de $53-100 \%$. Sería importante determinar en otro estudio los motivos del inicio de vida sexual temprana y los conocimientos de riesgo y uso de anticonceptivos que tengan, así como la fuente de estos conocimientos y su efectividad. Es necesario implementar una política de estado para la educación en sexualidad con enfoque ético, moral, humano, biológico y científico, de acuerdo a cada grupo etario y de desarrollo.

\section{Agradecimientos}

El estudio fue realizado gracias a las Dras. Dora Estripeaut y Elizabeth Castaño del Hospital del Niño, el Dr. Aurelio Núñez del Programa Nacional de ITS/VIH/SIDA y a la Sra. Martha Icaza y la Dra. Edilma Berrio de UNFPA-PANAMA. Deseamos agradecer a la sección de Salud Sexual y Reproductiva del Instituto Conmemorativo Gorgas de Estudios en Salud, en especial a la Dra. Ruth De León por permitirnos utilizar la base de datos ENASSER 2009. Igualmente al programa de Measure Evaluation de Tulane University / USAID / PEPFAR y al programa de excelencia profesional de USFSENACY'T (University of South Florida - Secretaria Nacional de Ciencia, Tecnología e Innovación, de donde las Dras. Ilenia Forero y Lorna Jenkins son ex-becarias) por las oportunidades de fortalecimiento técnico

\section{Declaración de los autores}

El resultado de la investigación y el contenido de la publicación son responsabilidad de los autores, no refleja la postura final de las instituciones para las cuales laboran.

\section{Referencias}

Alcarraz C, Arias K, Peña C, Ventura J, Calle M. (2008). Factores asociados a la realización de una prueba de detección de VIH en estudiantes universitarios. CIMEL, 13(2), 53-61.

Banco Mundial. (2013). Disponible en, http://data.worldbank.org/country/panama

Carrasco-Garrido P, López de Andrés A, Hernández Barrera V, Jiménez-Trujillo, I, Santos-Sancho J, and Jiménez-García R. (2011). Predictors of contraceptive methods among adolescents and young women residing in Spain. J Sex Med, 8, 2431-2438. 
Clifton J, Brice L, Wiggins-Smith J, Penrose L, Farooqi N. (septiembre, 2013). Influence of Sex Education on Condom Knowledge and Use Proficiency among Texas College students. Contraception, 88(3), 473.

De la Cruz-Peñarán D, Langer-Glas A, Hernández-Prado B, González-Rengijo GF. Conocimientos y actitudes de la pareja hacia la práctica de la planificación familiar en la Selva del Perú. (2003). Salud Publica Mex, 45, 461-471. Disponible en, http://www.insp.mx/salud/index.html

Diccionario de la Real Academia de la Lengua Española (DRAE). (2014). Disponible en, http://www.rae.es/recursos/diccionarios/drae

Fetis G, Bustos L, Lanas F, Baeza B, Contreras J, Hebel E, Marucich C. (2010). Factores asociados al uso de anticonceptivos en estudiantes de enseñanza media de la comuna de Temuco. Rev Chilena de Obstetricia y Ginecología, 73(6), 362-369.

González E, Caba F, Molina T, Sandoval J, Meneses R. (2003). Factores personales asociados al uso de anticonceptivos en adolescentes mujeres solteras sexualmente activas. CEMERA. Disponible en, http://www.cemera.uchile.cl/sogia/docs/2003/x2factores.doc

González M. Patrones de uso de anticonceptivos en diversos tipos de clínicas conforme la edad y género de los participantes, Programa de Planificación Familiar Título X del Recinto de Ciencias Médicas de la Universidad de Puerto Rico: 2007-2008. CIDE digital, 2(1), 53-74.

Instituto Nacional de Estadísticas y Censo (INEC), Contraloría General de la República de Panamá. Definiciones y Explicaciones utilizadas para el Censo de 2010. Disponible en, http:/ / www.contraloria.gob.pa/inec/archivos/P3561Definiciones.pdf

Instituto Nacional de Estadísticas y Censo (INEC), Contraloría General de la República de Panamá. Contraloría General de la República de Panamá (CG). (2011).

Instituto Nacional de Estadísticas y Censo (INEC), Contraloría General de la República de Panamá. Contraloría General de la República de Panamá (CG). (2012).

Joint United Nations Programme on HIV/AIDS (UNAIDS). (2013). Global AIDS Report 2013.

León F, Roca S, Ríos A. (2000). La Consejería Balanceada aumenta la calidad de la atención y el conocimiento de las mujeres sobre anticonceptivos. Population Council. Disponible en, http://www.popcouncil.org/pdfs/frontiers/FR.final

Organización Mundial de la Salud (OMS). Repositorio de datos. 2014. Disponible en, http://apps.who.int/gho/data/?theme=main 
Invest. pens. crit.

Vol. 2, No. 5, septiembre-diciembre 2014.

pp. $51-62$

Organización Panamericana de la Salud (OPS). Generador de tablas. 2013. Disponible en, http://www1.paho.org/English/SHA/coredata/tabulator/newTabulator.htm

Reis M, Ramiro L, Gaspar M, Alves J. (diciembre, 2012). Nationwide survey of contraceptive and sexually transmitted infection knowledge, attitudes and skills of university students in Portugal. Int J Clin Health Psychol 2013, 13, 127-137.

Schiappacasse V, Vidal P, Casas L, Dides C \& Díaz S. (diciembre, 2003). Chile: Situación de la saludy derechos sexuales y reproductivos. Santiago de Chile.

Taylor T, Hembling J, Bertrand J. (2014): Ethnicity and HIV risk behaviour, testing and knowledge in Guatemala, Ethnicity \& Health, DOI:10.1080/13557858.2014.893562.

Trejo-Ortiz P, Moreno-Chávez P, Macías-Aguilar M, Valdez-Esparza G, Mollinedo F, Lugo L, Araujo R. (noviembre, 2011). Conocimiento y comportamiento sexual en adolescentes. Área Académica Ciencias de la Salud. Universidad Autónoma de Zacatecas. Revista Cubana de Enfermería, 27(4), 273-280.

Vázquez A, Suárez N. (mayo-agosto, 2006). Conocimiento y uso de métodos anticonceptivos en la población de 15 a 44 años del área urbana, Cunduacán. Tabasco 2005. Horizonte Sanitario, 5(2).

World Economic Forum (WEF). (2013). The Global Competitiveness Report 2012-2013. Disponible en, http://www.weforum.org/reports/global-competitiveness-report-2013-2014 